\title{
A Survey on Routing Techniques of Data-Centric Wireless Sensor Networks
}

\author{
Nitika Vats Doohan \\ Institute of Engineering and Technology, DAVV, \\ Khandwa Road, Indore, M. P., INDIA
}

\author{
Sanjiv Tokekar \\ Institute of Engineering and Technology, DAVV, \\ Khandwa Road, Indore, M. P., INDIA
}

\begin{abstract}
Wireless Sensor Network (WSN) is a network of spatially distributed, autonomous sensors nodes deployed for various kinds of monitoring applications. To enable reliable communications between sources to destination, many routing protocols have been proposed in the past by many researchers. These protocols are designed considering inherent sensor nodes constraints i.e. limited battery backup, memory and computation capacity. Generally, routing protocols in WSNs have been classified on the basis of their route determination techniques such as proactive, reactive and hybrid. Further routing protocols classification is done on the basis of querybased techniques. This paper briefly discusses the various routing algorithms and few concurrent open research issues are also summarized.
\end{abstract}

\section{General Terms}

Network, Protocols, Routing.

\section{Keywords}

Sensor nodes, WSNs.

\section{INTRODUCTION}

A sensor is a device that produces a reckonable response to a change in a physical condition, such as temperature, pressure and weight etc. Sensors are used to measure basic physical phenomena including acceleration, shock, vibration, humidity, flow rate, force, magnetic field and wind speed. Normally the sensor node consists of sensing unit (convert analog signal to digital signal), processing unit (process the information gathered), transceiver unit (transmit and receive data signal) and powered by batteries. There are various types of sensors available and their deployment largely depends to satisfy the application requirements.

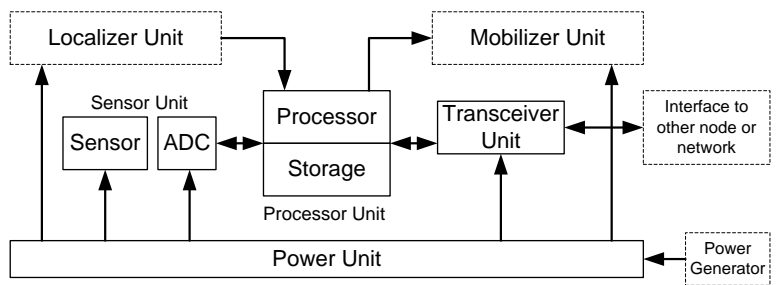

Figure 1: Block schematic of a sensor node.

A wireless sensor network (WSN) consists of large number of densely deployed sensors which cooperatively monitor the environmental conditions. Sensor produces a measurable response to the monitored physical condition. In wireless sensor network, each autonomous sensor route the information to the sink. The sensor nodes powered by the batteries are normally neither changeable nor rechargeable. There are other constraints in WSN for instance their bandwidth and routings Basic architecture of wireless sensor network consists of three basic things i.e. stimulus, sensor node and sink. Stimulus is the event for monitoring intended physical conditions occurred at the sensor node. Sensor nodes are micro-electro-mechanical systems (MEMS) which are powered by batteries having data processing \& communication capabilities $[1,2]$. Sensor node senses the event, collects and processes the data and finally route it to the destination node called sink. Generally, sensor node collect data intermittently and forward collected data upon request. Sink is the information collection center where all sensor nodes send their data for meaningful processing.

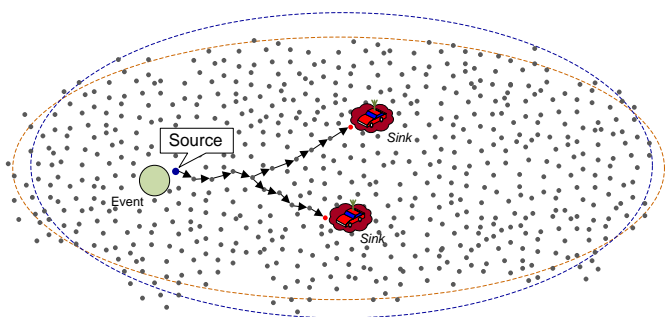

Figure 2: Sensor Network Model

Figure 2, shows network of nodes with two mobile sinks. Sinks are application specific and can be single or multiple. Sink can be stationary or mobile depending upon the application. When an event has occurred the nearest node act as a source and will transmit the sensed data to the sink. The aggregated data reached to the sink. The most distinguish short coming of stationary sink is that the sensor nodes which are in the vicinity of the sink will drain with their resources swiftly resulting overall reduced network lifetime. Hence, mobile sink can results in increased network life-time of WSN.

WSNs are widely used for monitoring application such as military surveillance, health care and event tracking etc. WSN also found to be extremely useful in security applications including intrusion detection, criminal hunting, health diagnosis and continuous monitoring of the patient. Sensor nodes can be deployed by air-drop in the area of interest and found very promising for rescue operations such as locating survivors, identifying the high risk area and minimizing the personnel involvement usually in dangerous scouting missions such as in case of landmine sniffing [2-3]. Sensor nodes are used in smart homes and offices for controlling temperature, moisture and air flow in the different parts of building. Algorithm scalability, limited computational power, storage 
capacity are the important design criterion for sensor network due to huge number of nodes in a wireless sensor network.

As compare to the ad-hoc network, sensor network is more prone to failure as the battery usually neither replaceable nor rechargeable. The energy of sensor network drained swiftly. Sensor networks are data-centric and having the feature of data aggregation / data fusion which helps in reducing the consumption of bandwidth and power.

\section{ROUTING}

Data dissemination is the process of routing the data from source to destination. Sensor nodes sense the triggered event and then route the data to the intended node known as sink. The way route is established from source to sink is referred as routing. This route can be modified by preferring the shortest route or the strongest route or deselecting the weaker or longer route. Routing is the process of sending the data from one node to another. A routing protocol is a convention or standard which controls how the nodes decide which way to route the packets. Route stability is an important optimization factor. A variety of routing protocols has been proposed in recent past. Routing in the sensor nodes is completely different in comparison to traditional routing. WSN architecture is a multi-hop communication system in which data is send via multi hops to the destination. The major challenges that a routing protocol designed for WSNs faces are mobility of nodes and resource constraints.

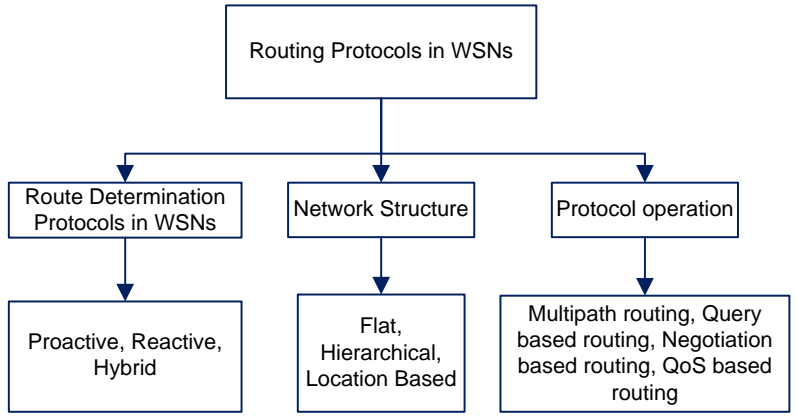

Figure 3: Classification of various WSNs routing protocols

Due to the above described problem, the routing is one of the main problems in Wireless Sensor Networks.

\section{Characteristics for a routing protocol}

We define the characteristics for the routing protocols that shall lead to the better and efficient design of sensor networks [4]

(i) Fault-tolerant and distributed- a fault-tolerant and distributed sensor network shall be able to sustain the non-stop operations all the time.

(ii) Adaptive to change according to the mobility of nodesthe network shall be able to adapt changes when the sensor nodes move due to the requirements.

(iii) Loop independent- There shall be a loop free configuration in order to avoid deadlocks.

(iv) Localized

(v) Reliable by using multiple paths- the multiple paths shall be desirable owing to the requirements of fault-tolerance.

(vi) Scalable in large-scale sensor networks- the network shall have a provision of dynamic scalability that shall be in line with the adaptability.

\subsection{Data-Centric Routing Protocols in Wireless Sensor Networks}

In data-centric routing protocol, whenever a sink requires any data it sends a query message to the different part of the sensor network field. After receiving this query message sensors node replies and sends data to the sink. In data-centric protocol attribute based naming is used which specifies the properties of the data. Many researchers worked and proposed the different versions of data-centric protocols.

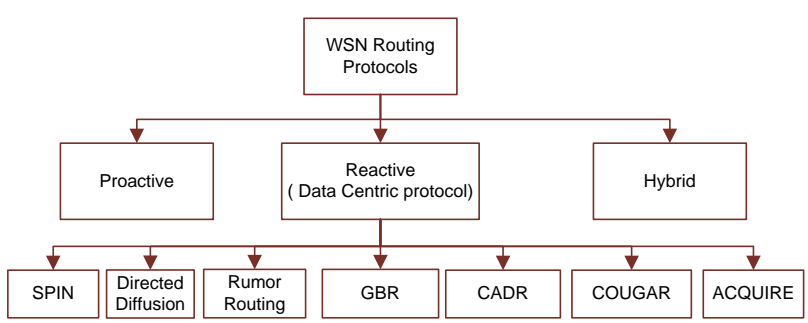

Figure 4: Classification of Routing Protocols

\subsubsection{Flooding}

Flooding [5] is a technique used for routing in sensor networks through the broadcasting. Whenever any sensor node receives a data, it broadcasts this message to all its neighbors, then the receiving neighbors repeat the same process until the message reached to the destination i.e. sink. There are certain problems associated with flooding approach specifically implosion, overlapping, and resource blindness. In case of data implosion, node receives same copies of data, more than one times means there is duplicity of messages.

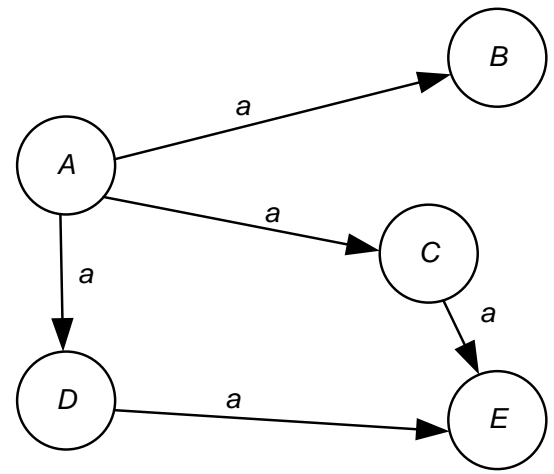

Figure 5: The implosion problem

As shown in figure 5, Node A floods its data to all of its neighbors. Here, node $\mathrm{E}$ gets two identical copies of same data eventually. In overlapping, the same event is sensed by more than one node due to overlapping regions of coverage where as the resource blindness is caused due to many redundant transmissions.

\subsubsection{Gossiping}

Gossiping [6, 7] is the customized version of flooding approach. In Gossiping, whenever any node receives a message it forwards this packet to randomly selected neighbors and when the selected neighbors get this message they will again select the random neighbors and forward this message. This process continues till the data reached to the destination. Gossiping has a problem like overlap in which two sensors cover same geographic region and send the data to same sensor by which it get the redundant data. 


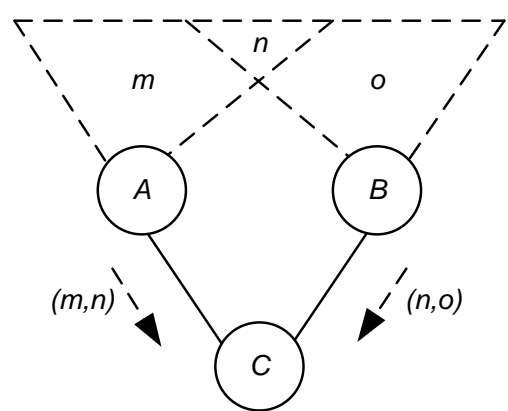

Figure 6: Node $\mathrm{C}$ gets the copy of data from nodes $\mathrm{A}$ and $B$ as they are geographically overlapped.

\subsubsection{SPIN}

Sensor Protocols for Information via Negotiation (SPIN) [8, 9] is another variant of data centric protocol designed to overcome the problem of flooding and gossiping. It uses data negotiation and resource-adaptive algorithms to eliminate the redundant data transmission of throughout the network occur during flooding [5]. In SPIN, when an event occurs, sensor node broadcast the advertisement message which contains high-level data descriptor, called meta-data, to its neighbors. If the neighbor is interested in data, then on receiving this advertisement it sends a request to the sender for data. As shown in figure 7, Node A starts by advertising its data to node B. Node B responds by sending a request to node A. After receiving the requested data, node $\mathrm{B}$ then sends out advertisements to its neighbors, who in turn send requests back to $\mathrm{B}$.
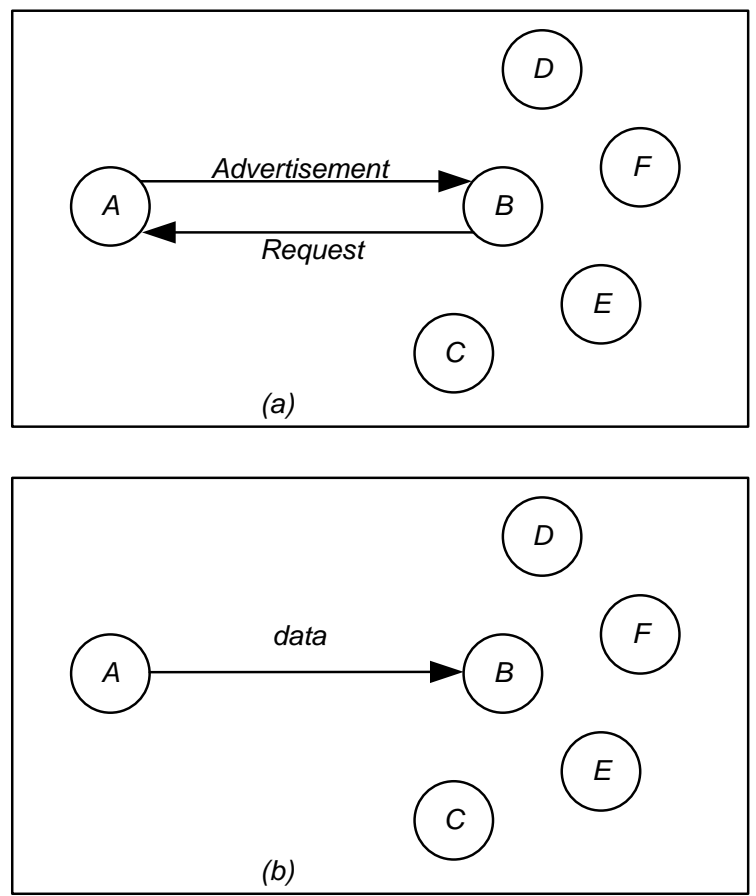

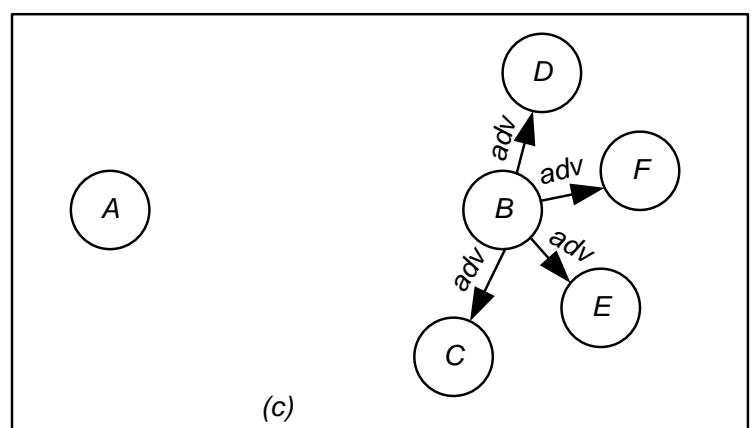

Figure 7: SPIN protocol

The main shortcoming with the SPIN is that it doesn't guarantee the delivery of the data packets. Therefore it can't be used for applications that require guaranteed or reliable delivery of data packets.

\subsubsection{Directed Diffusion}

Directed Diffusion [11] is an application-aware protocol uses on-demand data query and send data for delivery to inquiring destination. In directed diffusion, the sink sends the interest and whole network works on attribute-value pair. Each sensor node stores the interest which carries the entry of timestamp and gradient fields. Once the interest is propagated, the gradients are set up from source to sink. Whenever the source gets the data, it will send it to the sink. The directed diffusion is an on-demand data centric protocol. However, directed diffusion is not suitable for the applications where continuous flow of data is required. There are three different phases of directed diffusion:

1. Interest propagation.

2. Data propagation.

3. Reinforcement.

Interest is propagated in the network with the help of neighbours by the sink. Then the nodes generated the link known as gradient and reply back to the node from where the interest is generated. Hence, multiple paths generated to send the data but only one path is selected for routing the data. Once the path is set, data can be sent from source to destination.

Various variants of directed diffusion are proposed by the many researchers. These routing algorithms which improve the working of directed diffusion, they are as follows:

1. Directed diffusion using passive clustering

2. An energy aware broadcast scheme for directed diffusion

3. Node's credit based directed diffusion for wireless sensor network

4. RDDLA [19]

Vlado Handziski et al [16] propose a new strategy for improving the energy efficiency of a directed diffusion. They proposed that in directed diffusion if we divide the network in the passive clusters having each cluster a cluster node and different number of cluster members. By this clustering, overhead of energy expenditure in interest diffusion and exploratory data messages is reduced to certain extent.

CAO Zhi-yan et al [17] proposed an energy aware broadcast scheme for directed diffusion. They design a simple and efficient broadcast scheme. The proposed scheme has different phases:

1. Distance Estimation: In distance estimation, a node calculates the distance of node from which it receives a packet. For this, it first checks the strength of the 
received signal which associating with power of received signal and estimates the distance from the received power using free space wireless propagation models.

2. Energy and distance aware segment delay: In the second phase, this approach a random delay is inserted before rebroadcast based on remainder energy and distance.

3. Size and slot of delay window: In third phase of proposed algorithm, size and slot of delay window is determined by the network density.

4. Counter based and distance based rebroadcast suppression: In this phase redundant rebroadcasts are reduced.

The above four phases compose of simple broadcast scheme. Energy and distance aware segment delay mechanism enhances the efficiency of the broadcast reduce the collision and balance the energy consumption. Redundant rebroadcasts are reduced by counter based and distance based rebroadcast suppression.

Farnaz Dargahi et al [18] proposed a Node's credit based directed diffusion for wireless sensor networks, where the limitations of nodes are removed. The node just chooses a neighbor from which it last receives the event without knowing it battery status for message passing. For solving this problem, Farnaz enhances the knowledge of node using five factors they are:

1. Vs (Number of successful or unsuccessful transmission)

2. VE (Residual energy in candidate node battery)

3. VB (Traffic load at each node)

4. VR (Distance of node to a sink)

5. VC (How many source can cover by each node)

The first parameter (Vs) is the number of successful or unsuccessful transmission and how nodes succeed to deliver packets in the past. A low amount of this parameter of a node means that the node failed to route message in the past. This parameter is increased with each successfully routed packet and decrease with each failing in routing packets.

The second parameter (VE) is the residual energy in candidate node's battery. Eq. 1 is used for calculating this parameter. This parameter has an important effect on increasing the network lifetime.

$V E=\frac{\text { existing energy }}{\text { capacity of battery }}$

And the third parameter (VB) is traffic load at each node This parameter is computed according to Eq. 2 as:

$\mathrm{V}_{\mathrm{B}}=\frac{\text { free space of buffer }}{\text { all space of buffer }}$

If there would be high traffic load in candidate node, end to end delay will increase in sink node, in addition to this high traffic load will cause more spending energy in candidate node. The forth parameter (VR) is the distance of candidate node to destination (sink). Interest message of sink node is able to count and record the number of hops that passes through it. This allows nodes to discover the neighbor with minimum number of hops to the sink. The fifth factor (VC) is how many sources can cover by each node, when the exploratory data pass through a node the node will record such source ID's. The number of these sources ID represents how many sources are covered by this node.

A. Sayyad et al [19] has proposed a region directed diffusion in sensor network using learning Automata. They divided sensor network in some regions using bully algorithm. In this, the nodes which are close to each other are in one region. After this, we find an optimum path for transmitting data to a sink and for this it uses learning automata. Learning Automaton has a finite set of actions and each action has a certain probability of getting rewarded by the environment of the automaton.

\subsubsection{Rumor Routing}

Rumor routing (RR) is another variant of Directed diffusion approach. It lies in between the flooding and query flooding approach. RR generally suited for a network application where the events are less. In rumor routing whenever there is any event occurs at the node; an agent is generated and event is flooded in the local table and then information is propagated with the help of agent [7]. RR works with a single path i.e. from source to destination. For large events, cost of an agent is also increasing and hence RR is preferred when there is small event.

\subsubsection{Gradient Based Routing}

Gradient-Based Routing is another modified version of the Directed Diffusion in this each node can find the minimum number of hops to the sink, and it is called height of the node. In GBR [7], gradient is the difference between a node's height (minimum number of hopes to sink) and its neighbor. The packet is forwarded to the link, which has the largest gradient.

There are 3 different data spreading techniques:

1. Stochastic Scheme: If there are two or more next hops has same gradient, then the node are chosen on random basis.

2. Energy-based scheme: The node will not participate in the transmission when its energy goes below to a threshold value.

3. Stream-based scheme: In this scheme if the node is busy then it diverts the new streams away from it.

\subsubsection{Constrained Anisotropic Diffusion routing protocol}

M. Chu et al [13] have proposed a CADR protocol, a variant of data-centric protocol. Constrained Anisotropic Diffusion is a generalized form of directed diffusion. In this protocol the main theme is to query the sensors and route data in a network in order to maximize the information gain, while minimizing the network bandwidth and latency. This is possible by activating those sensors which are close to a particular event and dynamically adjusting data routes. The major difference from Directed Diffusion is the consideration of information gain in addition to the communication cost. This protocol use two types of techniques they are: Information-driven sensor querying (IDSQ), Constrained anisotropic diffusion routing (CADR).

\subsubsection{COUGAR}

Y. Yao and J. Gehr [14] have proposed a data-centric routing protocol named COUGAR. In this, the sink generates a query plan which specifies the necessary information about the data flow and in-network computation for the incoming query. In this, a leader is selected which is responsible for the necessary flow of data and data aggregation. In COUGAR, when leader node aggregates the data it just sends it to the sink.

The below table shows the different routing protocols with the problem associated with them. 


\begin{tabular}{|c|c|c|}
\hline $\begin{array}{c}\text { Routing } \\
\text { Protocols }\end{array}$ & $\begin{array}{c}\text { Data } \\
\text { aggregation }\end{array}$ & \multicolumn{1}{|c|}{ Problem Associated } \\
\hline Flooding & $\mathrm{P}$ & Implosion \\
\hline Gossiping & $\mathrm{P}$ & $\begin{array}{c}\text { Overlap problem } \\
\text { continues flow of the data is } \\
\text { required. }\end{array}$ \\
\hline $\begin{array}{c}\text { Directed } \\
\text { Diffusion }\end{array}$ & $\mathrm{P}$ & $\begin{array}{l}\text { Unable to handle large number } \\
\text { of events }\end{array}$ \\
\hline $\begin{array}{c}\text { Rumor } \\
\text { routing }\end{array}$ & $\mathrm{P}$ & $\mathrm{P}$ \\
\hline $\begin{array}{c}\text { Gradient- } \\
\text { Based }\end{array}$ & $\mathrm{P}$ & $\begin{array}{l}\text { 1. We have to dynamically } \\
\text { maintain a leader node to avoid } \\
\text { failure. } \\
\text { 2. Extra overhead to sensor nodes } \\
\text { by introducing additional query } \\
\text { layer on each sensor node. } \\
\text { 3. For data computation it } \\
\text { requires synchronization. }\end{array}$ \\
\hline COUGAR & $\mathrm{P}$ \\
\hline ACQUIRE & $\mathrm{N}$ & $\begin{array}{l}\text { If d is equal to network size, then } \\
\text { the protocol behaves similar to } \\
\text { flooding }\end{array}$ \\
\hline
\end{tabular}

Figure 8: Data-Centric routing protocols

\section{Conclusion}

Routing in sensor networks is very promising research issue. As sensor nodes are constrained of energy, computational capability, storage capacity. Therefore it is preferred that a node should participate in communication whenever deemed necessary/ asked to do so. A list of data centric protocols is shown in figure 8 with associated deficiencies. Many researchers have worked in this area. The paper gives general overview about routing protocols developed under data centric approach. It will help the researchers to better understand the work done in this specific area.

\section{REFERENCES}

[1] Akyildiz I F, Su W, Sankasubramaniam Y, "Wireless Sensor Networks: A Survey," In the Proceedings of Computer Networks, Vol. 38, March 2002, pp. 393-422.

[2] Akkaya K, Younis M, "A Survey on Routing Protocols for Wireless Sensor Networks," In the Proceeding of Adhoc Network Elsevier, September 2003, pp. 325-349.

[3] Hogler K, Andrew W, "A Short Survey of Wireless Sensor Networks," In the Proceedings of Telecommunication Networks Group, October 2003.

[4] Holos G, Starosweicki M, Aitouche A, "Optimal Design of Fault Tolerant Sensor Network," In the Proceedings of IEEE International Conference on Control Application, September 2000, pp.467-472.

[5] W. Heinzelman, J. Kulik, and H. Balakrishnan, "Adaptive protocols for information dissemination in wireless sensor networks," in the Proceedings of the 5th Annual ACM/IEEE International Conference on Mobile Computing and Networking (MobiCom'99), Seattle, WA, August 1999.

[6] S. Hedetniemi and A. Liestman, "A survey of gossiping and broadcasting in communication networks," Networks, Vol. 18, No. 4, pp. 319-349, 1988.
[7] D. Braginsky and D. Estrin, "Rumor Routing Algorithm for Sensor Networks," in the Proceedings of the First Workshop on Sensor Networks and Applications (WSNA), Atlanta, GA, October 2002.

[8] Shen C, Srisathapornphat, Jaikaeo C, "Sensor Information Networking Architecture and Applications," In the Proceedings of IEEE pers. Communication, August 2001, pp.52-59.

[9] D. Estrin et al., "Next century challenges: Scalable Coordination in Sensor Networks," in the Proceedings of the 5th annual ACM/IEEE international conference on Mobile Computing and Networking (MobiCom'99), Seattle, WA, August 1999.

[10] C. Schurgers and M.B. Srivastava, "Energy efficient routing in wireless sensor networks," in the MILCOM Proceedings on Communications for Network-Centric Operations: Creating the Information Force, McLean, VA, 2001

[11] C. Intanagonwiwat, R. Govindan, D. Estrin, "Directed diffusion: A scalable and robust communication paradigm for sensor networks", in the Proceedings of the 6th Annual ACM/ IEEEInternational Conference on Mobile Computing and Networking (MobiCom'00), Boston, MA, August 2000.

[12] Bulusu N, "Scalable Coordination for Wireless Sensor Networks: Self-configuring Localization Systems", In the Proceedings of ISCTA, 2001, pp.1-6.

[13] M. Chu, H. Haussecker, and F. Zhao, "Scalable Information-Driven Sensor Querying and Routing for ad hoc Heterogeneous Sensor Networks," The International Journal of High Performance Computing Applications, Vol. 16, No. 3, August 2002.

[14] Y. Yao and J. Gehrke, "The cougar approach to innetwork query processing in sensor networks," in SIGMOD Record, September 2002.

[15] N. Sadagopan et al., "The ACQUIRE mechanism for efficient querying in sensor networks," in the Proceedings of the First International Workshop on Sensor Network Protocol and Applications, Anchorage, Alaska, May 2003.

[16] Vlado Handziski, K. Frank and D., "Improving the Energy Efficiency of Directed Diffusion Using Passive Clustering", in the Proceedings of the EWSN 2004, LNCS 2920, pp 172-187, Springer-Verlag Berlin Heidelberg 2004.

[17] CAO Zhi-yan, JI Zhen-Zhou, HU Ming-Zeng, "An energy-aware broadcast scheme for directed diffusion in wireless sensor network", in the Proceedings of the Journal of Communication and Computer, Volume 4,ISSN1548-7709,USA, May 2007.

[18] Farnaz Dargahi, Amir Masoud Rahmani, Sam Jabehdari, "Nodes Credit based Directed Diffusion for wireless sensor networks," in the Proceedings of the International journal of Grid and Distributed Computing, 2007.

[19] A.Sayyad, M. Shojafar,Z. Delkhah and A. Ahamadi, "Region Directed diffusion in Sensor Network Using Learning Automata: RDDLA", in the Proceedings of the Journal of Advances in Computer Research.pp7183,2011 . 